Whang, Y.C. J. geophys. Res. 82, 1024-1030(1977)

Jackson, D. J. \& Beard, D. B. J. geophys. Re's. 82, 2828-2836 (1977)

1. Russell, C. T. Geophys. Res. Lett. 3, 125- $129(1976)$
2. Russell, C. T. Geophys. Res. Lett. 3, 589-592(1976)

13. Dolginov, Sh. Sh., Zhuzgov, L. N., Sharova, V. A., Buzin, V. B. \& Yeroshenko, Ye. G. The Magnetosphere of Venus (in Russian) preprint No. 19(193).65 (IZMIRAN Moscow 1977) Dolginov, Sh. Sh., Yershenko, Ye. G. \& Zhuzgov, L. N. J. geophys. Res. 81, 3353 (1976)

5. Russell, C. T. Geophys. Res. Lett. 5, 81 (1978).

6. Russell, C. T. Geophys. Res. Lett. 5,85 (1978)

Brown, L. W. Astrophys. J. Lo't. 198, L.89- L.92(1975)

8. Freeman, J. W. in Proc. NATO Adranced Study Institule on Origin of the Solar Sistem (in the press).

9. Slattery, W. L. Icarus 32, 58-72(1977)

20. Elphic, R. C. \& Russell, ( T. Geophys. Re's. Le'tl, (in the press)

\section{Uranus and the shape of elliptical rings}

AN interesting question raised by the discovery ${ }^{1-3}$ of rings around Uranus concerns the ellipticity of the $\varepsilon$ ring, the outermost of the five rings detected. When the star SAO158687 passed behind the Uranus system (with a closest approach to the centre of the planet of about $25,000 \mathrm{~km}$ ) its light was occulted twice by the $\varepsilon$ ring. The first part of the ring to occult was about $100 \mathrm{~km}$ wide and $51,700 \mathrm{~km}$ from the planet's centre; the second part was about $40 \mathrm{~km}$ wide and $51,050 \mathrm{~km}$ from the centre $^{1}$ (J. L. Elliot, personal communication). These two points were separated in angle by about $120^{\circ}$. The average transmission of starlight during these events, $35 \%$ and $10 \%$, respectively, lead to optical depths that are, within experimental error, inversly proportional to the two widths (J. L. Elliot, personal communication), strongly suggesting that there is the same amount of material in the rings at the two points sampled. Here we account for the variable width of the ring by differences in the orbital eccentricities of the individual particles composing the ring.

A narrow elliptical ring must be composed of particles in elliptical orbits with their major axes in nearly perfect alignment. As the alignment becomes less perfect, the ring width fattens and becomes less elliptical, until complete randomisation of axis directions results in a circular ring.

Partial misalignment of orbital axes could cause the different ring widths observed, but there is another possibility. Consider an elliptical ring as shown in Fig. 1, composed of particles with perfectly aligned orbital axes. Particle 1 is on the outer edge of the ring at pericentre, particle 2 is on the inner edge, and orbit 2 has a slightly different eccentricity from orbit 1 . The rest of the ring material is in orbits between 1 and 2 . The orbits of particles 1 and 2 are described by the expressions for confocal ellipses:

$$
\begin{aligned}
& \text { Orbit } 1 r_{1}=r_{1 p}\left(1+e_{1}\right) /\left(1+e_{1} \cos \theta\right) \\
& \text { Orbit } 2 r_{2}=r_{2 p}\left(1+e_{2}\right) /\left(1+e_{2} \cos \theta\right)
\end{aligned}
$$

where $r_{1 p}$ and $r_{2 v}$ are the pericentre distances, $\theta$ is the polar angle measured from pericentre, and $e_{1}, e_{2}$ are the eccentricities. The ring width at pericentre is $\Delta r_{\mathrm{p}}=r_{1 \mathrm{p}}-r_{2 \mathrm{p}}$.

The two points of the $\varepsilon$ ring observed during the passage of SAO158687 differ in their distance to the centre of Uranus by

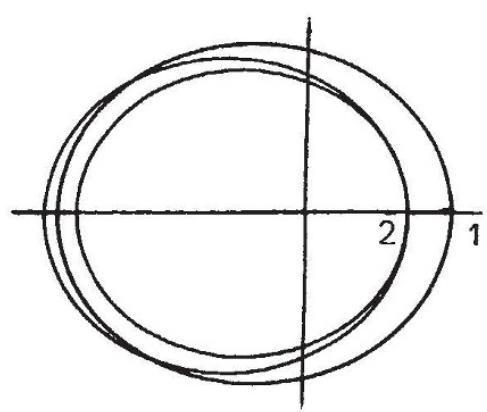

Fig. 1 Illustrating orbits at the edges of a ring. Two possible orbits for particle 2 are shown. about $600 \mathrm{~km}$. This sets a lower limit on the eccentricity of the ring of about $e=0.008$. The two observed points, plus the assumption that the $\varepsilon$ ring is never closer to Uranus than the next inner ring (the $\delta$ ring, at a radius of $48,400 \mathrm{~km}$ ) imply an upper limit to the eccentricity of $e \approx 0.13$. This assumption should be valid if the rings are truly coplanar, but is suspect otherwise.

Equations $(1 a)$ and $(1 b)$ can be combined and expanded to first order in $e$ to yield

$$
\Delta r=r_{1}-r_{2} \approx \Delta r_{\mathrm{p}}+\Delta r_{\mathrm{p}} e_{1}(1-\cos \theta)-r_{1 \mathrm{p}} \Delta e(1-\cos \theta)
$$

where $\Delta r$ is the radial spacing between the two orbits as a function of $\theta$ (as the orbits are not circles, this is not exactly the same as the width of the ring, but the difference is everywhere $<10 \%$ for $e \leqslant 0.4$ ), and $\Delta e=e_{2}-e_{1}$.

If the term $\Delta r_{\mathrm{p}} e_{1}(1-\cos \theta)$ in equation (2) is ignored (although it can make a significant contribution if $e$ is as large as the 0.13 value mentioned above), we see that if $r_{1 p}=51,000 \mathrm{~km}$, $\Delta e$ need be only as large as 0.0005 to give ring width variations of the order of $50 \mathrm{~km}$. If $\Delta e \geq \Delta r_{\mathrm{p}} / 2 r_{1 \mathrm{p}}$, then $\Delta r=\mathrm{O}$ at at least one point around the ring. Presumably, a ring width of zero would be quickly broadened by interparticle collisions. How much broadening would occur and whether this applies to Uranus' rings is an open question.

According to equation (2), a broad variety of ring shapes are possible. The narrowest point can be at the pericentre $(\Delta e<0)$, or the apocentre $\left(\mathrm{O}<\Delta e \leqslant \Delta r_{\mathrm{p}} / 2 r_{1 \mathrm{p}}\right)$, or anywhere in between $\left(\Delta e>\Delta r_{\mathrm{p}} / 2 r_{1 \mathrm{p}}\right)$. In the last case, of course, there must be two narrow points located directly across the major axis from each other.

With only a single observation of the rings, the model cannot be appreciably constrained, but it does predict that ring width should vary smoothly around the ring in a way that can be fitted by equation 2 . More observations, such as those suggested by Liller $^{4}$, are eagerly awaited.

Code 685,

R. L. LuCKE

Goddard Space Flight Center,

Greenbelt, Maryland 20771

Received 21 November; accepted 14 December 1977.

1. Elliott, J. L., Dunham, E. \& Mink, D. Nature 267, 328-330 (1977).

3. Hubbard, W. B., Coyne, G. V., Gehrels, T., Smith, B. A. \& Zellner, B. H. Nature 268, 33-34 (1977).

4. Liller, Wm. Astrophys. J. 82, 929 (1977).

\section{$\lambda$-Transition between normal and superfluid ${ }^{4} \mathrm{He}$ in the high-speed rotating frame}

THE rotating helium cryostat (1-m diameter, 3,000 r.p.m.) at Southampton provides a novel way of studying the behaviour of liquid helium. The centrifugal acceleration fields (up to $5,000 \mathrm{~g}$ at the periphery) enable pressure differences up to 20 bar to be generated in radial ducts containing liquid helium between the axis and the periphery of the rotor. We have recently rotated the cryostat at temperatures down to $1.8 \mathrm{~K}$, well below the $\lambda$ transition to superfluid helium. The $\lambda$-transition temperature is suppressed by increasing pressure so that the rotor becomes a unique tool for the study of the phase transition boundary between normal helium $\mathrm{He}(\mathrm{I})$ and superfluid helium $\mathrm{He}(\mathrm{II})$. Here we report observations of a step-like temperature difference of several tens of millikelvin across the boundary. The temperature jump can be explained in terms of the positive value of the volume coefficient of expansion for $\mathrm{He}(\mathrm{I})$ over a finite temperature interval above the $\lambda$-line, and the presence of a non-convecting intermediate state of finite thickness separating the two phases. 